\title{
Characteristics of 3D Printed Plastic Scintillator
}

\author{
Dong-geon Kim, Sangmin Lee, Junesic Park, Jaebum Son, Yong Hyun Kim, and Yong Kyun Kim \\ Hanyang University, Nuclear Engineering, Seoul, South Korea
}

\begin{abstract}
Digital Light Processing (DLP) 3D printing technique can be a powerful tool to fabricate plastic scintillator with a geometrically desired shape in innovatively fast time. Plastic scintillator with the size of $30 \mathrm{~mm} \times 30 \mathrm{~mm} \times 10 \mathrm{~mm}$ was fabricated by using the plastic resin and the DLP 3D printer (ASIGA, Pico2 ${ }^{\mathrm{HD}}$ ). The characteristics of decay time, energy resolution, intrinsic detection efficiency were analyzed and compared between the fabricated 3D printing plastic scintillator and a commercial plastic scintillator BC408 (Saint-Gobain Crystal). Decay time profile of the tested plastic scintillators was measured for ${ }^{137} \mathrm{Cs}$ Compton maximum electron $477 \mathrm{keV}$ by using a modified time correlated single photon counting (TCSPC) setup. The time profile was fitted by reconvolution function, and each decay time component and contribution was analyzed. For energy resolution of plastic scintillator, the Gaussian spectrum for ${ }^{137} \mathrm{Cs}$ Compton maximum electron $477 \mathrm{keV}$ was selectively measured by using the $\gamma-\gamma$ coincidence experimental setup. As a result, it was confirmed that the 3D printing plastic scintillator showed average decay time $15.6 \mathrm{~ns}$ and energy resolution $15.4 \%$. These characteristics demonstrates the feasibility of 3D printing plastic scintillator as a radiation detector.
\end{abstract}

Index Terms - Digital Light Processing (DLP), 3D Printer, UV polymerization, Plastic Scintillator, Scintillation Performance

\section{INTRODUCTION}

$\mathrm{P}$ LASTIC scintillator has been developed with various designs to expand its applications in radiation detection field since the 1950s when plastic scintillator was first invented. These plastic scintillators can be commonly fabricated by thermal polymerization technique [1]. A liquid solution with scintillation components is polymerized in heater, depending on a temperature profile with time. However, this technique has some limitations: (1) its geometrically confined and simple shapes of rods, cylinder, flat sheets and (2) long fabrication time of one week or more.

In an effort to overcome these limitations of this thermal polymerization, 3D printing plastic scintillator has been studied [2], [3]. In our group, for the application to Digital Light Processing (DLP) 3D printing, the UV-cured plastic scintillator was developed using UV LED curing machine. It was confirmed that the characteristics of this UV-cured plastic

This work was supported by the National Research Foundation of Korea (NRF) grant funded by the Ministry of Science and ICT (No. NRF2016M2A2A6A03912636).

Dong-geon Kim is with the Nuclear Engineering Department, University of Hanyang, Seoul 04763, Korea (e-mail:kdgeon79@hanyang.ac.kr).

Sangmin Lee is with the Nuclear Engineering Department, University of Hanyang, Seoul 04763, Korea (e-mail: s6245299@hanyang.ac.kr). scintillator were relative light output $34 \%$ to BC408 (SaintGobain Crystal), transmittance 49\%, and decay time 2.46 ns [4]. Recently, a novel plastic scintillator with a new wavelength shifter and intermediate solvent was developed by DLP 3D printing technique, and its characteristics were relative light output $67 \%$ to BC408 and transmittance 56\% [3]. Although this 3D printing plastic scintillator showed excellent light output and transmission over the previous results, the demonstration for other main characteristics is required for practical use in radiation detection field.

In this work, for practical use, the characteristics of the $3 \mathrm{D}$ printing plastic scintillator were demonstrated. The characteristics of decay time and energy resolution were analyzed and compared with a commercial plastic scintillator BC408 (Saint-Gobain Crystal).

\section{MATERIALS AND METHODS}

$3 \mathrm{D}$ printing plastic scintillator with the size of $30 \mathrm{~mm} \times 30$ $\mathrm{mm} \times 10 \mathrm{~mm}$ was fabricated by the Digital Light Processing (DLP) 3D printer (ASIGA, Pico2 ${ }^{\mathrm{HD}}$ ). The material composition of plastic resin developed in the previous study [3] was used. Each experimental setup was constructed to measure and compare the characteristics of decay time and energy resolution for the 3D printing plastic scintillator (Sample) and commercial plastic scintillator (BC408). A modified time correlated single photon counting (TCSPC) setup was constructed to measure scintillation time profile. The measured time profile as shown in Figure 1 was fitted with reconvolution function to analyze each decay time constant and contribution.

In the case of energy resolution, it is difficult to measure the energy resolution since the photopeak is not found in the $\gamma$-ray energy spectrum due to low photoelectric cross section of plastic scintillators. In other to overcome this problem, we constructed the $\gamma-\gamma$ coincidence experimental setup and measured the Gaussian energy spectrum for the maximum Compton electron reaction (Compton edge) among several Compton electrons reacted with the scintillator as shown in Figure 2.

Junesic Park is with the Nuclear Engineering Department, University of Hanyang, Seoul 04763, Korea (e-mail: ergosphere84@gmail.com).

Jaebum Son is with the Nuclear Engineering Department, University of Hanyang, Seoul 04763, Korea (e-mail: jbs1000@hanyang.ac.kr).

Yong Hyun Kim is with the Nuclear Engineering Department, University of Hanyang, Seoul 04763, Korea (e-mail:kimyh04@naver.com).

Yong Kyun Kim is with the Nuclear Engineering Department, University of Hanyang, Seoul 04763, Korea (e-mail:ykkim4@hanyang.ac.kr). 


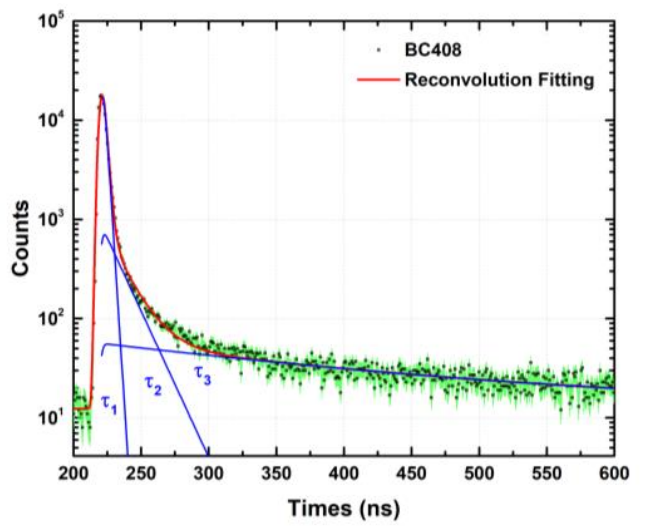

Fig. 1. Scintillation decay time profile of plastic scintillator $\mathrm{BC} 408$ for ${ }^{137} \mathrm{Cs}$ Compton maximum electron $477 \mathrm{keV}$.

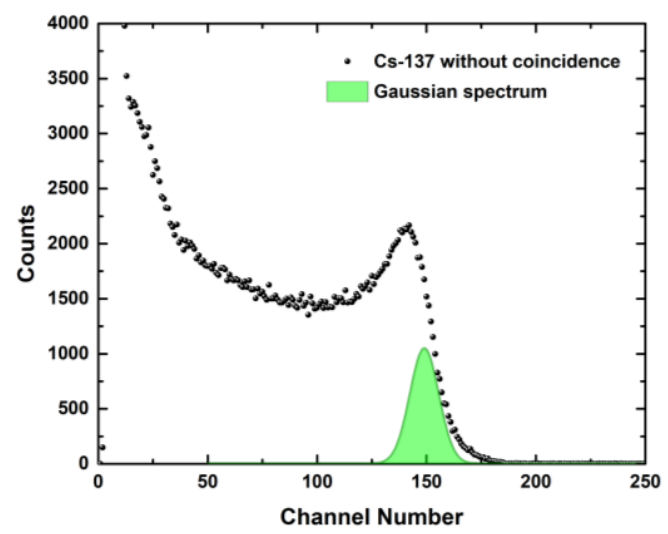

Fig. 2. ${ }^{137} \mathrm{Cs} \gamma$-ray pulse height spectrum (without coincidence spectrum) and Gaussian spectrum ( $\gamma-\gamma$ coincidence spectrum) for plastic scintillator BC408.

\section{RESULTS}

Table 1 shows the characteristics of plastic scintillators BC408 and Sample. It was confirmed that the average decay time 15.6 ns for Sample were compatible to BC408, which verifies that this $3 \mathrm{D}$ printing plastic scintillator can be used in high radiation environment of $\gamma$-ray. The energy resolution $15.4 \%$ of Sample was somewhat lower than $9.7 \%$ of BC408.

TABLE I

CHARACTERISTICS OF PLASTIC SCINTILLATORS

\begin{tabular}{lcc}
\hline \hline \multirow{2}{*}{\multicolumn{1}{c}{ Properties }} & \multicolumn{2}{c}{ Plastic scintillator } \\
\cline { 2 - 3 } & BC408 & Sample \\
\hline Peak wavelength $^{\text {a }}$ & $425 \mathrm{~nm}$ & $470 \mathrm{~nm}$ \\
Light output $^{\mathrm{a}}$ & $10,470 \mathrm{ph} / \mathrm{MeV}$ & $7,000 \mathrm{ph} / \mathrm{MeV}$ \\
Transmittance $^{\text {a }}$ & $76 \%$ & $56 \%$ \\
Average decay time $^{\text {Energy Resolution }}{ }^{\mathrm{b}}$ & $18.3 \mathrm{~ns}$ & $15.6 \mathrm{~ns}$ \\
\hline \hline
\end{tabular}

${ }^{a}$ These measurements are referred to J. Son et al. [3].

${ }^{b}$ This values represent the measurements at $477 \mathrm{keV}$ for Compton electron produced by ${ }^{137} \mathrm{Cs} \gamma$-ray source.

\section{CONCLUSION}

In this work, the characteristics of $3 \mathrm{D}$ printing plastic scintillator were analyzed for decay time, energy resolution and intrinsic detection efficiency. It was found that the average faster decay time $15.6 \mathrm{~ns}$ of $3 \mathrm{D}$ printed plastic scintillator than $18.3 \mathrm{~ns}$ of $\mathrm{BC} 408$. These characteristics demonstrates the feasibility of $3 \mathrm{D}$ printing plastic scintillator as a radiation detector.

\section{REFERENCES}

[1] C. H. Lee, J. Son, T. H. Kim, and Y. K. Kim, "Characteristics of Plastic Scintillators Fabricated by a Polymerization Reaction," Nucl. Eng. Technol., vol. 49, no. 3, pp. 592-597, 2017.

[2] Y. Mishnayot, M. Layani, I. Cooperstein, S. Magdassi, and G. Ron, "3D Printing of Scintillating Materials," no. June, pp. 1-4, 2014.

[3] J. Son, D. G. Kim, S. Lee, J. Park, Y. Kim, T. Schaarschmidt, and

Y. K. Kim, "Improved 3D Printing Plastic Scintillator Fabrication,"

J. Korean Phys. Soc., vol. 73, no. 7, pp. 887-892, 2018.

[4] S. Lee, J. Son, D. G. Kim, J. Choi, and Y. K. Kim,

"Characterization of plastic scintillator fabricated by UV LED curing machine," Nucl. Instruments Methods Phys. Res. Sect. A Accel. Spectrometers, Detect. Assoc. Equip., vol. 929, no. July 2018, pp. 23-28, 2019. 